\title{
Induction of CD4+CD25+Foxp3+ regulatory T cell response by glatiramer acetate in type 1 diabetes
}

\author{
Guoliang Cui ${ }^{1}$, Yuebo Zhang ${ }^{1}$, Zhenwei Gong ${ }^{1}$, Jingwu Z Zhang ${ }^{2}$, Ying Qin Zang ${ }^{1}$
}

${ }^{I}$ Key Laboratory of Nutrition and Metabolism, Institute for Nutritional Sciences, Shanghai Institutes for Biological Sciences, Graduate School of CAS, Chinese Academy of Sciences, 319 Yue Yang Road, Shanghai 200031, China; ${ }^{2}$ Institute of Health Sciences, Shanghai Institutes for Biological Sciences, Graduate School of CAS, Chinese Academy of Sciences, 319 Yue Yang Road, Shanghai 200031, China

Glatiramer acetate (GA) is an immunomodulatory peptide drug used to treat multiple sclerosis. Its treatment effect has been expanded to other autoimmune conditions such as uveoretinitis, inflammatory bowel disease, graft rejection and hepatic fibrosis. Here, we report that GA was effective in altering the clinical course of diabetes in cyclophosphamide (CY)-potentiated non-obese diabetic (CY-NOD) mice. Treatment with GA significantly reduced the diabetic rate in the mice and ameliorated insulitis, which coincided with increased CD4+CD25+Foxp3+ $T$ cell response in treated mice. GA treatment led to increased expression of transcription factor Foxp3 and elevated production of interleukin-4 (IL-4) both in vivo and in vitro. It was evident that the effect of GA on up-regulation of Foxp3 was mediated partially through IL-4. IL-4 was found to maintain Foxp3 expression and regulatory function of CD4+CD25+ regulatory $T$ cells (Tregs). This study provides new evidence that GA has treatment potential for type 1 diabetes through the induction of Tregs and that increased IL-4 production is partially responsible for the enhanced Treg's function in GA treatment.

Keywords: glatiramer acetate, regulatory T cell, Foxp3, type 1 diabetes

Cell Research (2009) 19:574-583. doi: 10.1038/cr.2009.12; published online 3 February 2009

\section{Introduction}

Glatiramer acetate (GA), also known as Copaxone, copolymer-1 or Cop-1, is a random copolymer of alanine, lysine, glutamate and tyrosine. GA has been used in the therapy of multiple sclerosis (MS) for over a decade [1-3]. Recently, GA has been investigated for other clinical indications, such as the treatment of uveoretinitis, inflammatory bowel disease, graft rejection and hepatic fibrosis [4-6]. Although the mechanism of action of GA is not well understood, according to various studies it is thought to involve in immunomodulation. Mechanisms potentially underlying the therapeutic effect of GA include MHC blockade, development of Th2-polarized GA-reactive CD4+ T-cells, deviation of Th1 to Th2

Correspondence: Ying Qin Zang

Tel/Fax: +86-21-54920913

E-mail: yqin@sibs.ac.cn

Recieved 22 November 2008; revised 27 November 2008; accepted 3 December 2008; published online 3 February 2009 immunity and type II macrophage-related modulation in both experimental animals and humans [7-9]. Treg constitutes a unique subset of $\mathrm{T}$ cells that play an important role in maintaining self-tolerance [10-12]. The transcription factor Foxp3 has been identified as a marker of Tregs and is critical for the development and function of Tregs [13-16]. Thus, augmentation of both the number and function of Treg represents a therapeutic opportunity for autoimmune conditions. Recent reports indicate that the deficiency in CD4+CD25+Foxp3+ Tregs in multiple sclerosis is restored by GA treatment. Hong et al. [17] reported that GA induced Tregs in both human and mouse immune systems in relevance to the treatment of MS. Jee et al. [18] demonstrated that GA treatment boosts the expression of Foxp3 on Tregs during EAE, and adoptive transfer of purified Tregs from GA-treated EAE mice is more effective in preventing EAE than those derived from untreated EAE mice.

Type 1 diabetes accounts for $5-10 \%$ of diagnosed cases of diabetes and is one of the most common autoimmune diseases. It is caused by loss of immune tolerance 
to self-antigen and subsequent $\mathrm{T}$ cell-mediated destruction of islet $\beta$ cells [19]. Injection of insulin is the first choice of treatment for type 1 diabetes. However, in later stages of type 1 diabetes many complications arise, including retinopathy, neuropathy and nephropathy, which unfortunately are not preventable by insulin injections [20]. Development of an immunomodulatory treatment may provide an alternative therapeutic option for this devastating disease. Since type 1 diabetes belongs to the category of autoimmune diseases, the inflammation of the islets may be dampened by GA treatment that is likely to induce Th1 to Th2 immune deviation and increased function of Treg. In this study, we investigated the role of GA in the treatment of type 1 diabetes and the underlying mechanism in cyclophosphamide (CY)-potentiated nonobese diabetic (CY-NOD) mouse model, an accelerated form of diabetes induced by injection of CY. The findings have important implications in the understanding of the regulatory mechanism of GA and in the treatment potential of GA in type 1 diabetes.

\section{Results}

GA administration improved type 1 diabetes in CY-NOD mice

We first examined the clinical effect of GA treatment in CY-NOD model. As shown in Figure 1, 25\% of the mice in the GA-treated NOD group became diabetic at 3 weeks after $\mathrm{CY}$ induction, while $83.3 \%$ of the mice were affected in the untreated NOD group (Figure 1A). Insulitis was significantly improved in GA-treated mice as compared with untreated controls. Stack graphic analysis of insulitic score showed that $31.6 \%$ of the islets from the GA-treated group were protected from lymphocyte infiltration, while only $3.2 \%$ of the islets in the untreated group remained free from insulitis (Figure 1B). The overall degree of insulitis markedly reduced in the GAtreated group. In addition, the treatment effect of GA was also evaluated under the same experimental conditions in the spontaneous NOD group and showed minimal effects (data not shown). These results suggest that GA administration improved type 1 diabetes in CY-NOD mice.

GA treatment upregulated the expression of Foxp3 and enhanced suppressive function of Tregs

As Tregs are critical to autoimmune inflammatory process, we examined whether GA treatment had an effect on Foxp3 expression in CY-NOD mice. CD4+ T cells were isolated from the mice with or without GA treatment, and foxp 3 expression was determined by real-time PCR and FACS analysis. The results revealed that foxp3 mRNA expression was increased in CD4+ T cells iso-
A
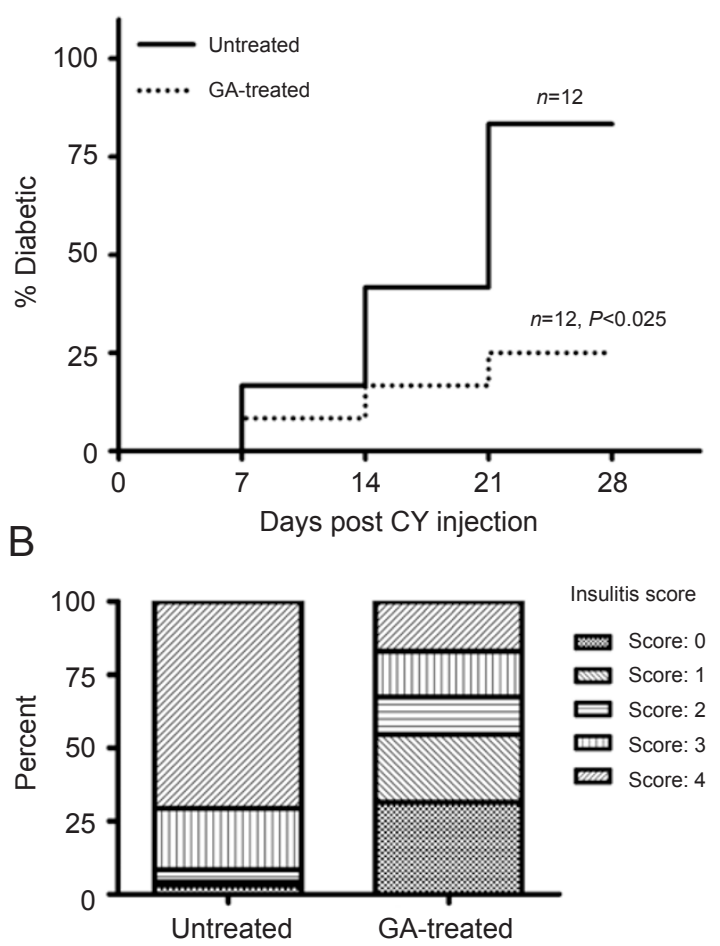

Figure $1 \mathrm{GA}$ treatment prevented the development of type $1 \mathrm{di}-$ abetes in CY-NOD mice. (A) 11-week old NOD mice were injected with GA (2 mg/mouse) every other day for a total of seven times before diabetes induction by CY ( $250 \mathrm{mg} / \mathrm{kg}$ body weight). $P$ value was calculated using log-rank test. (B) At 28 days after $\mathrm{CY}$ injection, mice were killed and islets were examined by $\mathrm{H} \& \mathrm{E}$ staining. The percentages of islets with various insulitis in the GA-treated and untreated groups are shown (100 islets/group).

lated from GA-treated mice as compared with those from untreated controls (Figure 2A). FACS analysis of intracellular Foxp3 staining showed that the Foxp3 expression in GA-treated mice was markedly increased in CD4+ $\mathrm{T}$ cells isolated from both splenocytes and pancreatic lymph nodes (Figure 2B). Although no significant differences in the total number of the spleen cells were observed between GA-treated and untreated groups (Figure 2C), it was evident that GA treatment led to an increased number of CD4+CD25+Foxp3+ cells from $0.79 \pm 0.22 \times$ $10^{6}$ to $1.26 \pm 0.16 \times 10^{6}$ in CY-NOD mice (Figure $\left.2 \mathrm{D}\right)(P$ $<0.05)$. CD4+CD25+ T cells isolated from GA-treated or untreated donor mice were adoptively transferred to the recipient NOD mice $24 \mathrm{~h}$ after $\mathrm{CY}$ injection. The purity of the transferred cells was at least $90 \%$ for CD4+CD25+ markers, and Foxp3 expression in these cells was higher than $70 \%$ as determined by flow cytometry (Figure 2E). All CY-NOD mice in which Tregs were transferred from 


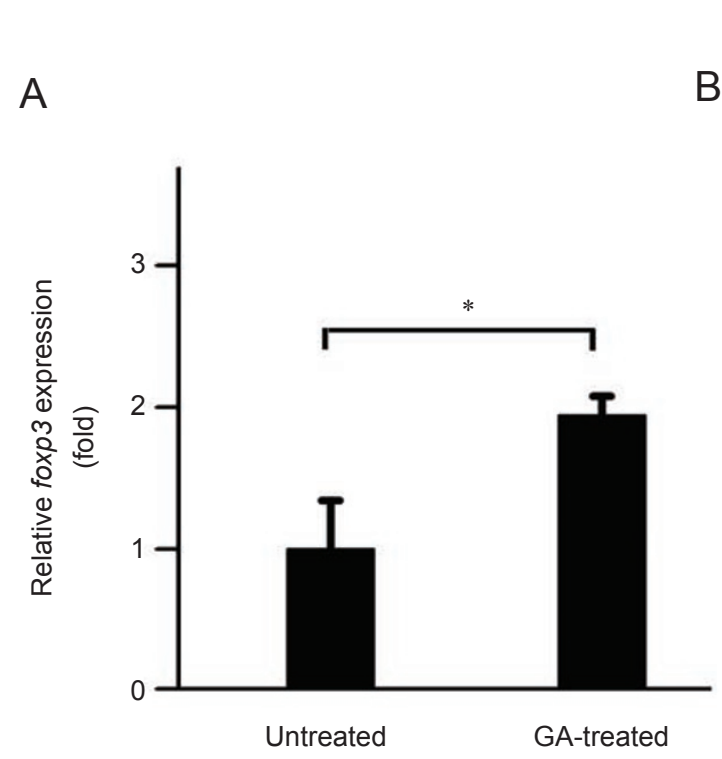

C

D

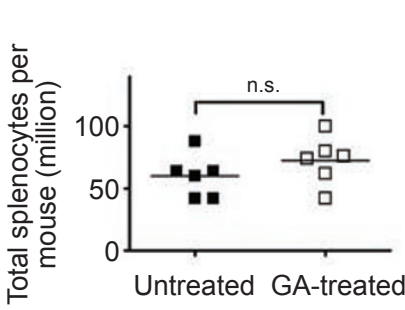

F

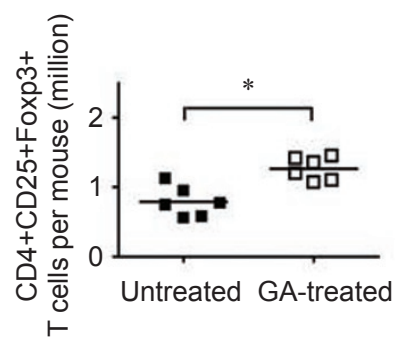

G
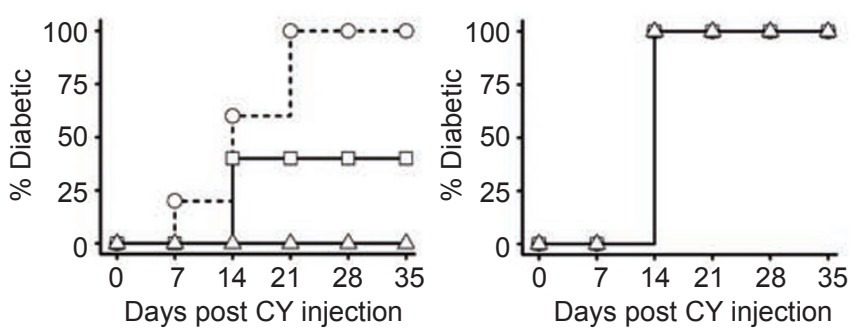

B

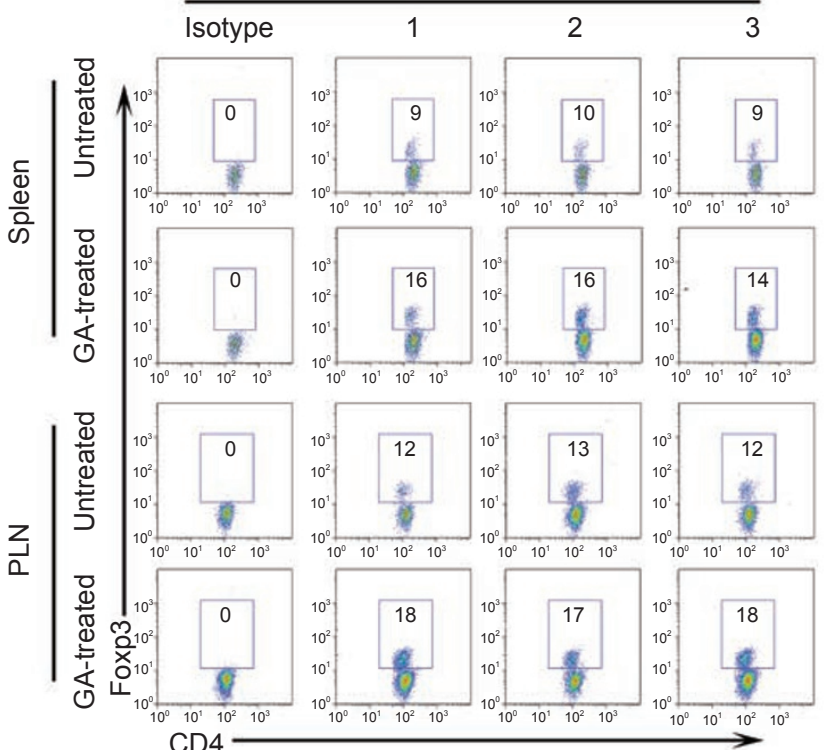

E

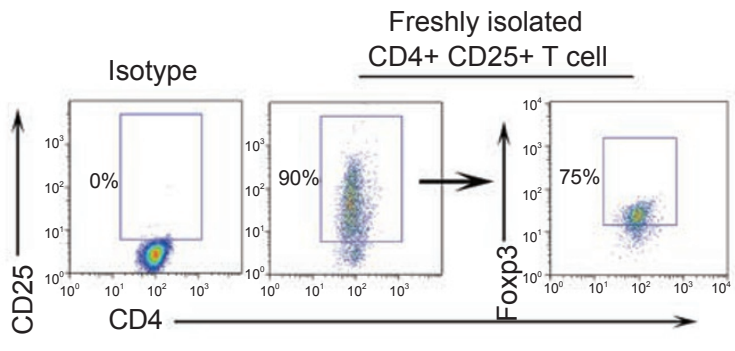

$\mathrm{H}$

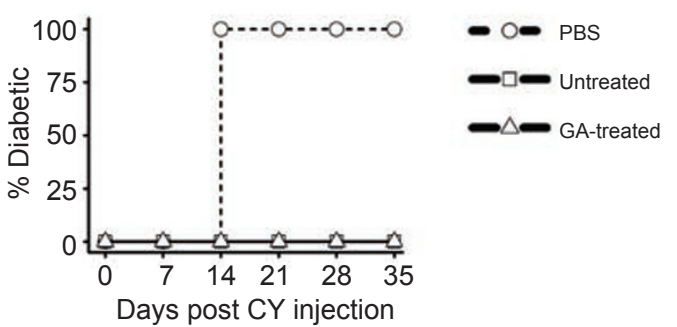

Figure 2 GA treatment increased the total number of Foxp3+CD4+CD25+ regulatory T cells. At 28 days after $C Y$ injection, NOD mice with or without GA treatment were killed. (A) CD4+ T cells were isolated and then subjected to real-time PCR $(n$ $=6$ mice/group, $* P<0.05)$. (B) Splenocytes and pancreatic lymph node (PLN) lymphocytes were stained with antibodies against CD4, CD25 and Foxp3. CD4+ T cells were gated and intracellular Foxp3 expression was shown above $(n=6$ mice/ group, representative data for three mice are shown above). (C) The numbers of total splenocytes are shown as above $(n=$ 6 mice/group, n.s., no significant difference). (D) The number of CD4+CD25+Foxp3+ T cells per mouse are shown as above ( $n=6$ mice/group, $* P<0.05$ ). (E) CD4+CD25+T cells were column-purified. Purity and Foxp3 expression were examined by flow cytometry assay. (F) CD4+CD25+ T cells from GA-treated or untreated mice were adoptively transferred to 11-week old recipient NOD mice $24 \mathrm{~h}$ after $\mathrm{CY}$ injection (one donor mouse corresponding to one recipient mouse, 5 mice per group). (G) $0.1 \times 10^{6} \mathrm{CD} 4+\mathrm{CD} 25+\mathrm{T}$ cells from GA-treated or untreated mice were adoptively transferred to 11 -week old recipient NOD mice $24 \mathrm{~h}$ after $\mathrm{CY}$ injection (4 mice per group). (H) $1.0 \times 10^{6} \mathrm{CD} 4+\mathrm{CD} 25+\mathrm{T}$ cells from GA-treated or untreated mice were adoptively transferred to 11-week old recipient NOD mice $24 \mathrm{~h}$ after $\mathrm{CY}$ injection (4 mice per group). Blood glucose level was measured weekly and considered to be diabetic when higher than $250 \mathrm{mg} / \mathrm{dl}$. 
GA-treated mice that had more CD4+CD25+ T cells $\left(1.45-1.91 \times 10^{6} /\right.$ mouse $)$ were protected from diabetes, while $40 \%(2 / 5)$ of mice in the control group that had less CD4+CD25+ T cells $\left(0.69-1.1 \times 10^{6} /\right.$ mouse) became diabetic (Figure 2F), indicating a correlation between the Treg number and diabetic rate. Figure $2 \mathrm{G}$ and $2 \mathrm{H}$ confirmed this correlation by showing significant protection when a high dose of Tregs were transferred to the CYNOD mice and no protection when a low dose of Tregs were used in the adoptive experiment, regardless of the donor cell origin.

Upregulation of Foxp3 mRNA and protein expression with GA treatment was also confirmed in an in vitro experiment when splenocytes isolated from NOD mice were cultured for 4 days in the presence of GA or mixed control peptides (Figure 3A and 3B). Intracellular staining of Foxp3 showed that GA treatment led to an increase of Foxp3 expression compared with the control peptides (Figure 3C). The inhibition of $\mathrm{T}$ cell proliferation assay showed that GA treatment was associated with enhanced suppressive properties of $\mathrm{CD} 4+\mathrm{CD} 25+\mathrm{T}$ cells compared with control peptides (Figure 3D). Our results indicate that GA treatment upregulated Foxp3-expressing cells and enhanced the suppressive function of Tregs, and that the protective effect of diabetic rate was related to the increased number of Tregs.

Induction of IL-4 by GA and the role of IL-4 in upregulation of Foxp 3 expression by GA

Previous studies indicated that GA administration promoted Th2 cytokine secretion in EAE mouse models and MS patients $[8,9]$. In this study, we asked whether GA had a similar effect on cytokine profile in this murine model of type 1 diabetes and whether cytokines induced by GA were responsive for upregulation of Foxp3. The in vitro cytokine profile of splenocytes isolated from NOD mice after 4 days of culture in the presence of GA or control mixed peptides showed an increase of IL-4 with GA treatment compared with control peptides (Figure 4A). In CY-NOD mice, the circulating level of IL-4 was significantly increased in GA-treated mice, while IL10, IFN $\gamma$ and TNF $\alpha$ levels were not significantly changed
A

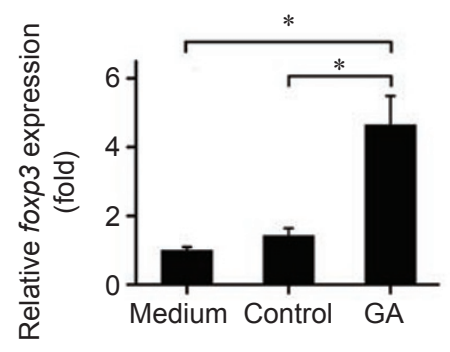

C

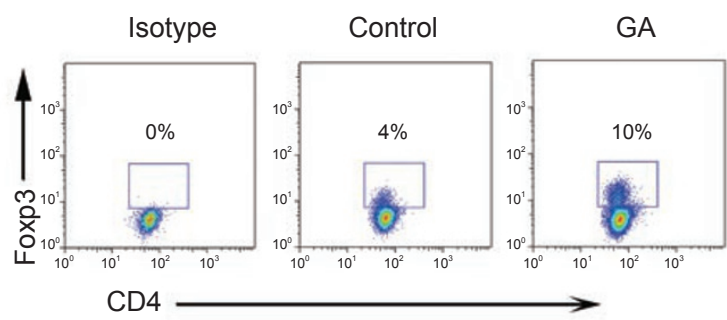

B

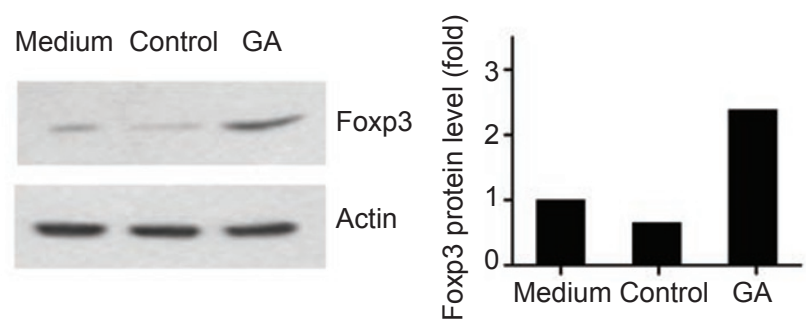

D

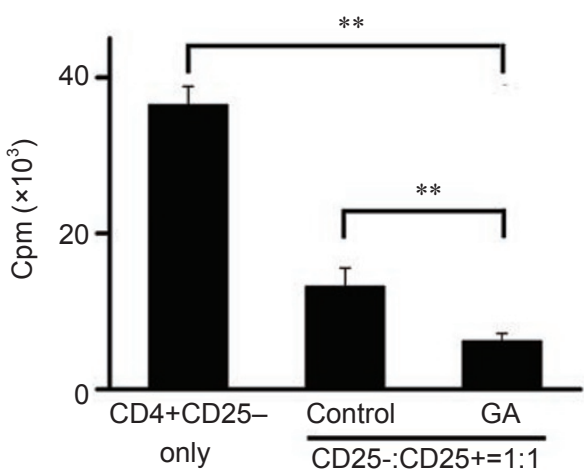

Figure 3 In vitro GA treatment upregulated Foxp3 expression and increased suppressive activity of CD4+CD25+ regulatory T cells. Splenocytes were isolated from 8-week old female NOD mice and cultured in medium only or in the presence of GA or control peptides for 4 days. (A) The Foxp3 expression level was examined by RT-PCR $(* P<0.05)$. (B) The Foxp3 expression level was also examined by Western blot and the optical density of the bands was quantified and normalized against that of actin. (C) After 4 days of culture, cells were collected and subjected to flow cytometry assay. The gate was set on CD4+ T cells to study intracellular Foxp3 expression. (D) CD4+CD25+ T cells were column-purified from control peptide-treated and GA-treated splenocytes and subjected to inhibition assay as described in Materials and Methods $(* * P<0.01)$. 

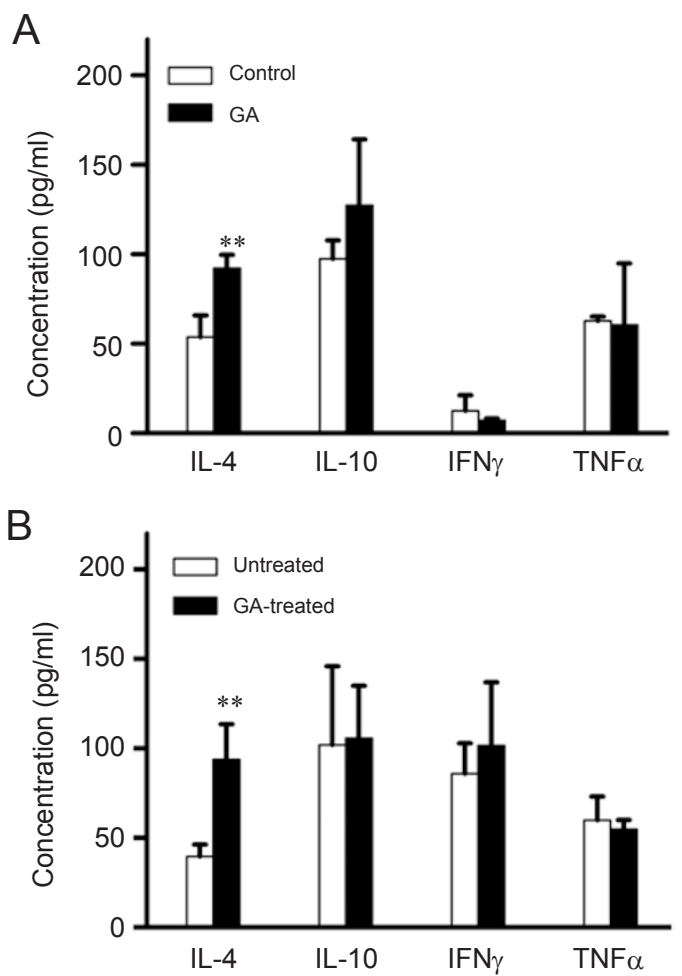

Figure 4 GA treatment promoted the secretion of IL-4. (A) Splenocytes were isolated from 8-week old female NOD mice and cultured in the presence of GA or control mixed peptides for 4 days. Then, the supernatant was analyzed by ELISA $(* * P<0.01)$. (B) At 28 days after $C Y$ injection mice were killed and serum was subjected to ELISA (6 mice per group; $* * P<0.01$ ).

\section{(Figure 4B).}

As GA treatment led to a significant increase of IL-4 level that correlated with a high level of Foxp3 expression, we next analyzed whether IL-4 played a role in the induction of Foxp3 expression by GA. First, we cultured total splenocytes together with GA or control mixed peptides for 4 days. Addition of an antibody directed at IL-4 partially reduced GA-induced Foxp3 expression, while an anti-IL-10 antibody did not affect Foxp3 induction by GA (Figure 5A). It was confirmed by intracellular staining of Foxp3 that GA treatment increased the expression of Foxp3, and that IL-4 blockade with IL-4 antibody aborted the effect of GA on Foxp3 expression (Figure $5 \mathrm{~B}$ ), indicating that IL-4 played an important role in this process.

IL-4 sustained Foxp 3 expression and suppressive activity of CD4+CD25+ T cells

Next we asked whether IL-4 treatment could upregulate Foxp3 expression. Splenocytes from NOD mice were cultured in the presence or absence of IL-4. In con- trast to a decline in Foxp3 expression of CD4+CD25+ T cells in the absence of IL-4, the addition of IL-4 led to sustained Foxp3 expression after 3 days of culture (Figure $6 \mathrm{~A}$, upper panel). We then addressed whether increased function of Treg was attributable to the generation of new Tregs by IL-4 or whether it was related to sustained Foxp3 expression by IL-4. To this end, CD4+CD25- T cells or CD4+CD25+ T cells were cultured in the presence of CD3/28 antibodies with or without IL-4 for 3 days. No change in Foxp3 expression was observed in the CD4+CD25- T cell group (Figure 6A, middle panel). However, Foxp3 expression decreased significantly in $\mathrm{CD} 4+\mathrm{CD} 25+\mathrm{T}$ cells as compared with the base level $(14 \%$ vs $76 \%)$ in the absence of IL- 4 , while the level was sustained at $31 \%$ in the presence of IL-4. Functional assay further suggested that CD4+CD25+ T cells treated with IL-4 had sustained regulatory functions compared with untreated controls (Figure 6B). These data indicated that IL-4 treatment led to sustained regulatory function through maintaining Foxp3 expression.

\section{Discussion}

In this study, we describe that GA treatment improves type 1 diabetes through induction of Tregs in CY-NOD mice. The conclusion is supported by the following experimental evidence: (i) the improved clinical course of diabetes correlates with increased Treg's functions; (ii) GA treatment selectively induces the production of IL-4; (iii) IL-4 plays an important role in sustaining expression of Foxp3 and maintaining Treg's functions.

Although a previous report suggested that NOD mice was not highly responsive to GA treatment [21] and our study has also shown to have minimal effects on spontaneous NOD mice, the treatment effect of GA was demonstrated in this study in CY-induced NOD mice having an acute inflammation state. The CY-induced diabetic model is apparently more susceptible to GA treatment and this may be related to the critical differences between the CY-NOD and spontaneous NOD models. Brode et al. [22] reported that $\mathrm{CY}$ accelerated diabetes development in NOD mice by decreasing Foxp3+ Tregs, and this process was accelerated compared with NOD mice. Our data supported the possibility that GA suppressed diabetes in CY-NOD mice through modulation of this process. Additionally, it is likely that the disease process in NOD mice takes much longer time, while GA's effect by the protocol used here could not be sustained long enough in terms of the Foxp3 expression and resulting immune tolerance. This may explain why GA is more effective in the CY-NOD mice.

In our adoptive transfer experiments, all of the recipi- 
A

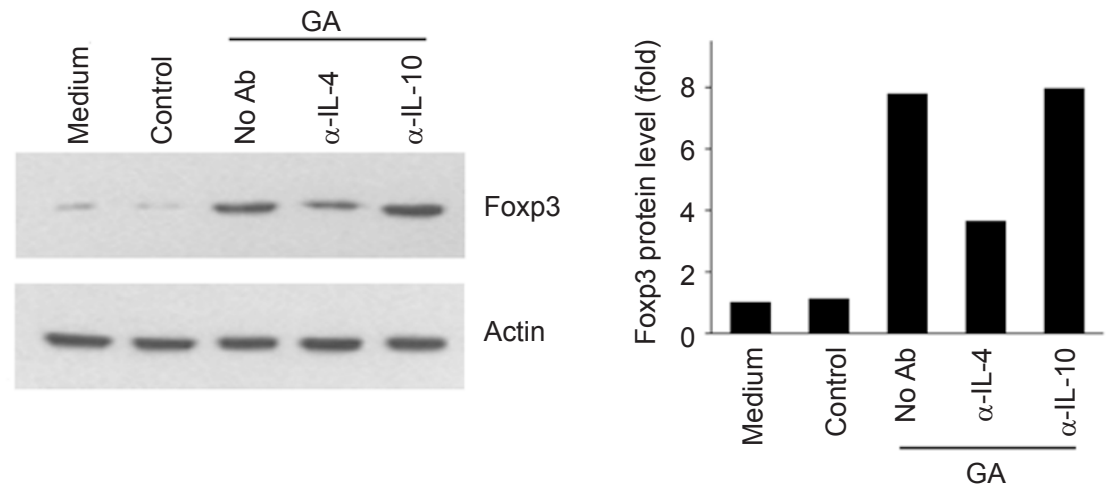

B

Isotype

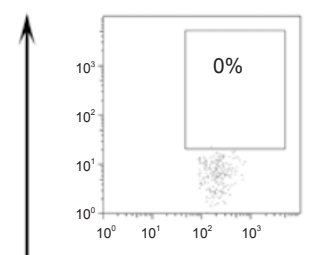

Control peptide

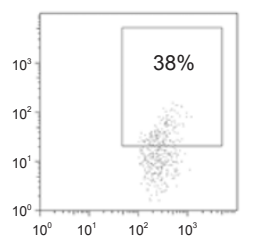

f
GA

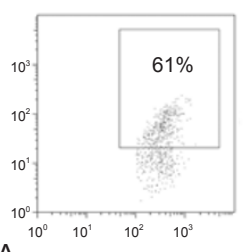

A
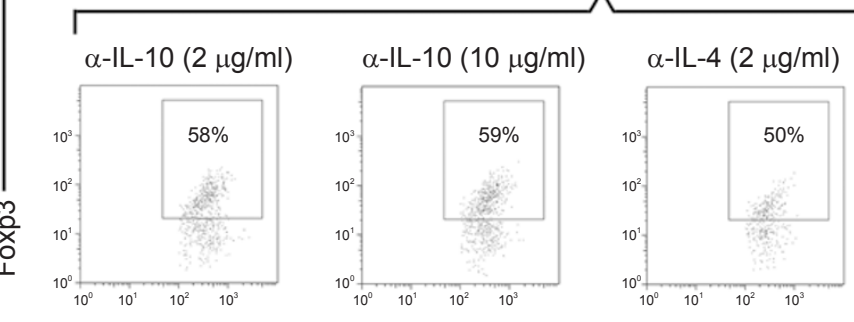

$\alpha-\mathrm{IL}-4(10 \mu \mathrm{g} / \mathrm{ml})$

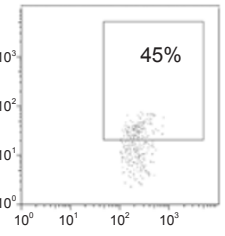

CD4

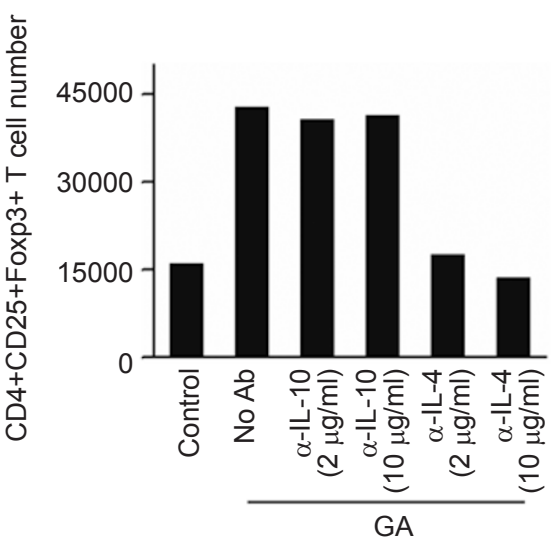

Figure 5 Upregulation of Foxp3 expression by GA was partially mediated by IL-4. (A) Splenocytes were isolated from 8-week old female NOD mice and cultured for 4 days in medium only, or in the presence of control mixed peptides or GA, or GA together with an anti-IL-4 or anti-IL-10 monoclonal antibody $(2 \mu \mathrm{g} / \mathrm{ml})$. The protein was extracted and subjected to Western blot. The optical density of the bands was quantified and normalized against that of actin. (B) $25 \times 10^{4}$ CD4+CD25- T cells column-purified from 8-week old female NOD mice were co-cultured with $25 \times 10^{4} \mathrm{~T}$ cell-depleted splenocytes in the presence of control peptide or GA or GA together with an anti-IL-4 or anti-IL-10 monoclonal antibody $(2 \mu \mathrm{g} / \mathrm{ml}$ or $10 \mu \mathrm{g} / \mathrm{ml})$. Cells were collected and stained with anti-CD4, anti-CD25 and anti-Foxp3 antibodies after 4 days. CD4+CD25+ T cells were gated, intracellular Foxp3 expression is shown in the upper panel and the total number of CD4+CD25+Foxp3+ T cells in the lower panel. 
A

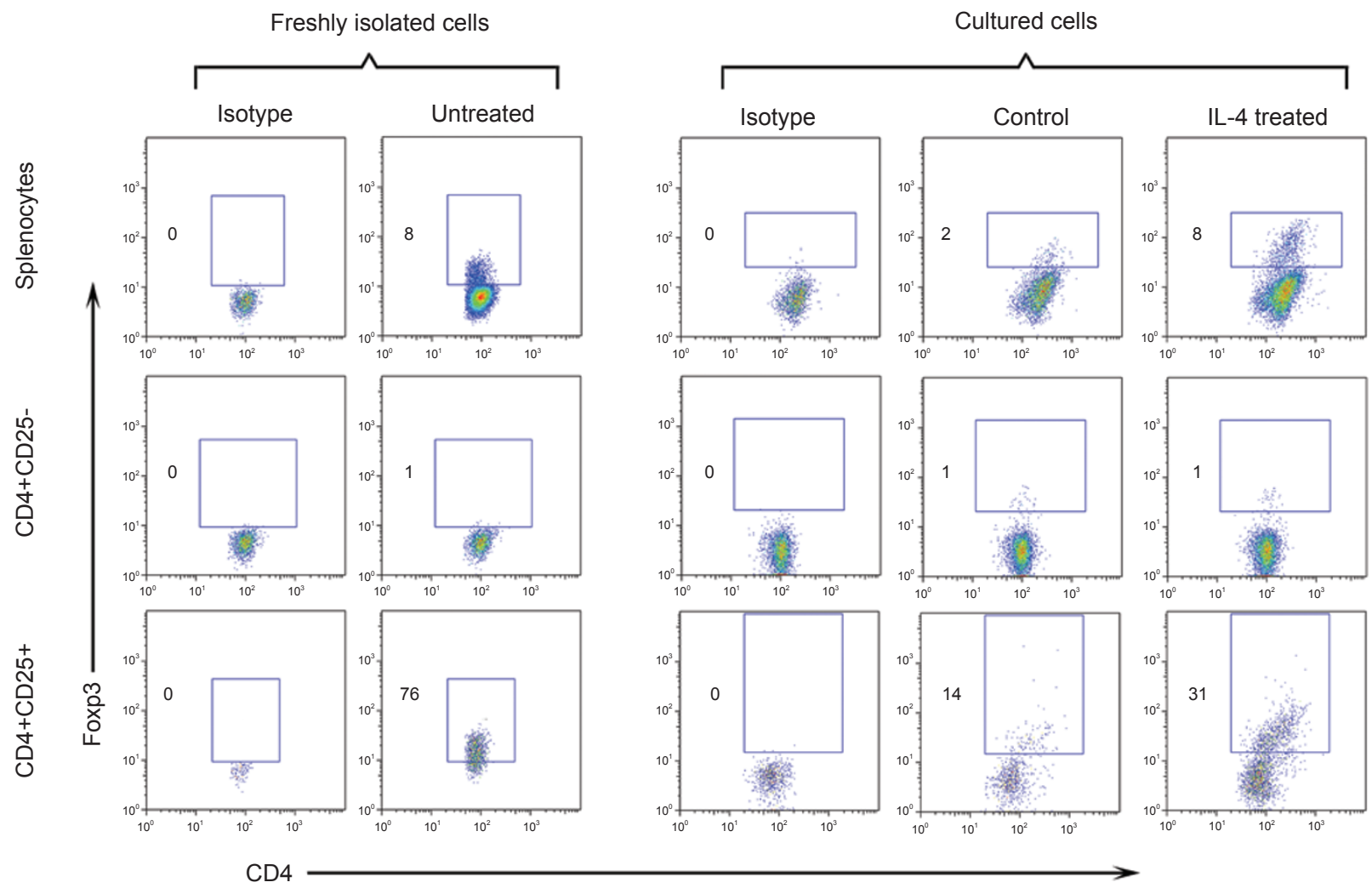

B

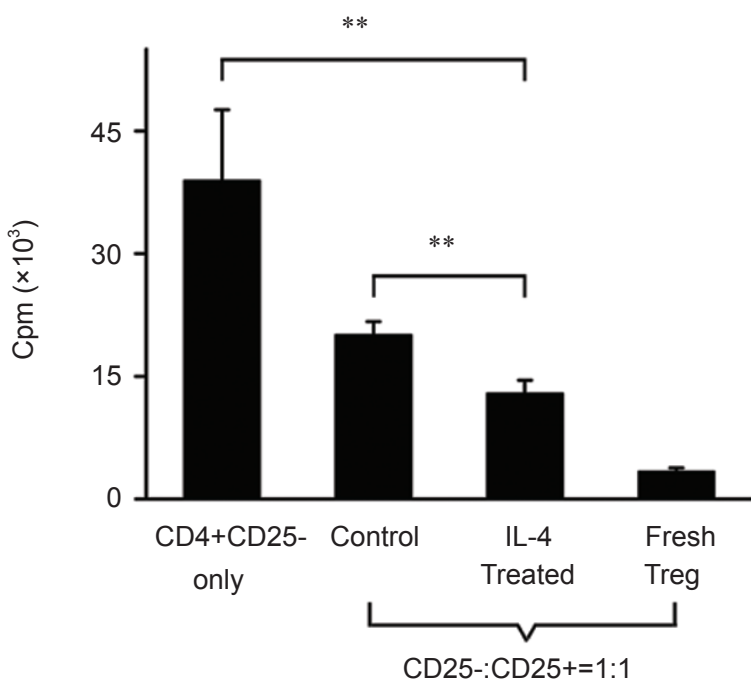

Figure 6 IL-4 maintained Foxp3 expression and suppressive function of CD4+CD25+ T cells. (A) Splenocytes, columnpurified CD4+CD25- T cells and CD4+CD25+ T cells from 8-week old female NOD mice were cultured with or without IL-4 $(20 \mathrm{ng} / \mathrm{ml})$ for 3 days in the presence of anti-CD3/CD28 antibodies as TCR stimulants $(2 \mu \mathrm{g} / \mathrm{ml})$. After 3 days, cells were subjected to FACS. CD4+ cell population was gated and Foxp3 expression is shown above. Freshly isolated cells were included as controls. (B) CD4+CD25+ T cells pre-treated with or without IL-4 for 3 days were washed and co-cultured with freshly isolated CD4+CD25- T cells and subjected to inhibition assay as described in Materials and Methods $\left({ }^{* *} P<0.01\right)$. 
ent mice that received the $\mathrm{CD} 4+\mathrm{CD} 25+\mathrm{T}$ cells derived from GA-treated mice were protected from $\mathrm{CY}$-induced diabetes, while $40 \%$ of the recipient mice that received $\mathrm{CD} 4+\mathrm{CD} 25+\mathrm{T}$ cells from untreated mice became diabetic. This might be explained by the notion that much more $\mathrm{CD} 4+\mathrm{CD} 25+\mathrm{T}$ cells are needed to survive in vivo and migrate to suppress effector $\mathrm{T}$ cells $[23,24]$. More CD4+CD25+Foxp3+ T cells were produced after GA treatment compared with the untreated mice among CY-induced NOD mice. Titration study with the same amount of cells transferred to recipients comfirmed that the total number of $\mathrm{CD} 4+\mathrm{CD} 25+\mathrm{T}$ cells is critical in protecting CY-induced NOD mice from diabetes.

In addition to evaluating the treatment effect of GA in diabetic models, much of the focus of this study was to explore the mechanism underlying the regulatory properties of GA. In this regard, we identified that GA treatment significantly promotes the regulatory function of Treg through cytokines, such as IL-4. Indeed, a number of studies have focused on the induction and expansion of CD4+CD25+ Tregs by cytokines. IL-2 [25-27], transforming growth factor $\beta$ (TGF $\beta$ ) [28-30] and IFN $\gamma$ [31] have been shown to be involved in the generation of Tregs. Moreover, Thornton et al. [27] reported that, when cultured in the presence of IL-2 and IL-4, CD4+CD25+ expanded without loss of suppressive functions. More recently, Skapenko et al. [32] reported that IL-4 could induce Foxp3 mRNA expression in CD4+CD25- T cell precursors.

Our findings are consistent with the previous studies that indicated upregulation of Foxp3 by GA treatment in both humans and mouse models $[17,18]$. However, the study here provides new insights into the molecular mechanism responsible for the increased expression of Foxp3. First, our results reveal that the increased expression of Foxp3 in CD4+CD25+ T cells correlates with the increased production of IL-4. The role of IL-4 in the induction of Foxp3 expression becomes apparent when a set of antibodies to various cytokines were tested. Second, we provide experimental evidence that the role of IL-4 is critical to the maintenance of the sustained expression of Foxp3 and regulatory function. It is likely that Foxp3 expression declines over time through spontaneous apoptosis [33]. Addition of IL-4 increased Foxp3 expression and maintained the suppressive property of Tregs. In this regard, the role of IL-4 differs fundamentally from the reported roles of other cytokines, TGF $\beta$ and IFN $\gamma$, in the induction of Treg. The modality of the other cytokines works through active conversion of CD4+CD25- T cells to Treg.

The role of IL-4 in the induction of Treg's function is particularly intriguing. It has been reported that IL-4 and IL-4R $\alpha$ knockout mice have normal Treg frequency with suppressive function similar to their wild-type littermates [32]. This discrepancy is reminiscent of the role of IFN $\gamma$ in peripheral conversion of CD4+CD25- T cells to Foxp3+ Tregs [31]. In that study, IFN $\gamma$ knockout mice had lower Foxp3 expression than that of wild-type mice during acute inflammation in EAE even though the expression of Foxp3 is normal in naïve state. In view of this, the roles of IL-4 and IFN $\gamma$ in the induction of Foxp3 expression and Treg function seem to differ from that of TGF $\beta$. CD4+CD25+ T cells from TGF $\beta 1$ knockout mice had abnormal Foxp3 expression and impaired suppressive function [30]. The discrepancy may suggest that TGF $\beta$ is essential for the generation of Tregs, while the role of IL-4 and IFN $\gamma$ is inducible by and associated with acute inflammation. In conclusion, our current study provides new insights into the role of GA in the treatment of type 1 diabetes and the mechanism of Treg functional maintenance through the action of IL-4.

\section{Materials and Methods}

\section{Animals}

11-week old NOD mice were purchased from the SLAX animal facility at the Shanghai Institutes for Biological Sciences. To synchronize and accelerate the development of diabetes, NOD mice were given a single intraperitoneal injection $(250 \mathrm{mg} / \mathrm{kg}$ body weight) of CY. Non-fasting blood glucose was measured every week with a glucose meter (Freestyle) and mice were considered diabetic when the blood glucose level was over $250 \mathrm{mg} / \mathrm{dl}$. In the treatment group, GA was administered ( $2 \mathrm{mg}$ per mouse) subcutaneously every other day for a total of seven times. All animal studies were conducted in accordance with the guidelines for animal studies issued by the Institute for Nutritional Sciences of Chinese Academy of Sciences.

\section{Peptides}

The control mixed peptide used in this study was a mixture of four peptides containing the four amino acids found in copaxone: peptide 1, SVNCAKKIV; peptide 2, QDKLTQWPKWLE; peptide 3, VYDFFVWL; peptide 4, APVIKARMMEYGTT. Copaxone was purchased from TEVA Pharmaceuticals.

\section{Histology}

Mice were killed and pancreata were dissected and immediately fixed in $4 \%$ paraformaldehyde. Paraffin-embedded 10 - $\mu$ m sections were stained with hematoxylin and eosin (H\&E) and examined under light microscope. Insulitis was scored following this standard as described previously [34]: score 0 , no lymphocytes infiltration; score 1, periductal infiltration; score 2, periinsulitis; score 3, intraislet infiltration; score 4 , severe destruction of islet and total loss of $\beta$ cells.

\section{Western blot}

Cells were lysed and protein was extracted and resolved by $10 \%$ SDS-PAGE. Immunoblot analysis was performed by trans- 
ferring proteins onto PVDF membranes (Millipore) using a Mini TransBlot apparatus (Bio-Rad). The membranes were blocked with $5 \%$ milk in TBST and incubated overnight at $4{ }^{\circ} \mathrm{C}$ with antibodies against Foxp3 (Santa Cruz) and Actin (Sigma) followed by incubation with HRP-conjugated secondary antibodies for $1 \mathrm{~h}$ at room temperature. Signals were detected by enhanced chemiluminescence (Pierce) according to the manufacturer's instructions.

\section{$R T-P C R$}

$1 \mu \mathrm{g}$ of RNA was reversely transcribed into cDNA using a random primer and an oligo (dT) primer (Takara), and $1 \mu \mathrm{l}$ of the synthesized cDNA product was then added into the PCR reaction mixture. Quantitative real-time RT-PCR was performed using an ABI Prism 7500 sequence detection system (Applied Biosystems). The sequences of the primers were as follows. foxp 3 forward primer: GGCAATAGTTCCTTCCCAGAGTT, foxp3 reverse primer: GGGTCGCATATTGTGGTACTTG; $\beta$-actin forward primer: TGACAGGATGCAGAAGGAGA, $\beta$-actin reverse primer: GTACTTGCGCTCAGGAGGAG. Gene expression was normalized to the level of $\beta$-actin in each example.

\section{Flow cytometry}

For surface staining of CD4 and CD25, T cells were resuspended in PBS containing 1\% bovine serum albumin (BSA) and were incubated on ice for $30 \mathrm{~min}$ with fluorochrome-conjugated antibodies (eBioscience) for these surface markers. For intracellular staining of Foxp3, cells were fixed and permeabilized and then stained with FITC-conjugated anti-Foxp3 mAb $\left(0.5 \mu \mathrm{g} / 10^{6}\right.$ cells; eBioscience). Stained cells were subsequently analyzed using a FACSAria or a FACS Calibur instrument (BD).

\section{Cell purification}

To isolate CD4+ T cells, splenocytes were prepared and depleted of B cells, macrophages, CD8+ T cells, NK cells, dendritic cells, erythrocytes and granulocytes using a mouse CD4+ negative selection kit (Dynal Biotech). To isolate CD4+CD25+ and CD4+CD25- T cells, purified CD4 $+\mathrm{T}$ cell populations were incubated with PE-labeled anti-CD25 antibody and anti-PE magnetic beads and isolated by MACS separation column (Miltenyi Biotec). Cell purity was assessed by FACS and was constantly higher than $90 \%$.

\section{ELISA}

ELISA kits for detection of murine IL-4, IL-10, IFN $\gamma$ and $\mathrm{TNF} \alpha$ were purchased from R\&D Systems, and were performed strictly following the manufacturer's instructions. Microtiter plates were coated overnight at room temperature with mouse monoclonal antibodies (capture antibody). Wells were then blocked for $1 \mathrm{~h}$ with PBS containing $1 \%$ BSA and washed four times with $0.05 \%$ Tween-20 in PBS. Duplicate standards and samples were added to adjacent wells and incubated for $2 \mathrm{~h}$ at room temperature. Wells were washed and detection antibodies were added. After $2 \mathrm{~h}$, wells were washed and incubated with an HRP-conjugated antibody in $1 \%$ BSA/PBS. Plates were washed four times and incubated for 30 min with the substrate $0.0125 \%$ tetramethylbenzidine $/ 0.008 \%$ $\mathrm{H}_{2} \mathrm{O}_{2}$ in citrate buffer $(\mathrm{pH}=5.0)$, and color development was stopped using $2 \mathrm{M} \mathrm{H}_{2} \mathrm{SO}_{4}$. Optical densities were measured using an ELISA reader, and concentration of cytokines was calculated using a double standard curve (R\&D Systems) in each plate.

\section{Suppression assay}

To determine the suppressive function of CD4+CD25+ T cells, $2 \times 10^{4}$ freshly isolated CD4+CD25- T cells (responder) were cultured in the presence or absence of CD4+CD25+ T cells. All cells were cultured for 3 days in the presence of Con A $(5 \mu \mathrm{g} / \mathrm{ml}$; Sigma) and $1 \times 10^{5}$ irradiated antigen presentation cells. [ $\left.{ }^{3} \mathrm{H}\right]$-thymidine incorporation during the last $16 \mathrm{~h}$ was measured as counts per minute $(\mathrm{cpm})$ using a $\beta$ plate counter.

\section{Adoptive transfer of regulatory $T$ cells}

$\mathrm{CD} 4+\mathrm{CD} 25+\mathrm{T}$ cells column-purified from the splenocytes of NOD mice with or without GA treatment were adoptively transferred to NOD mice $24 \mathrm{~h}$ after $\mathrm{CY}$ injection. PBS was injected as a control. Non-fasting blood glucose was monitored as described above.

\section{Statistics}

The log-rank test was used to calculate the difference between diabetes rate curves of NOD mice. Two-tailed Student's $t$-test was used to calculate differences in other assays. A $P$ value of less than 0.05 was considered statistically significant.

\section{Acknowledgments}

This work was supported in part by grants from the Hundred Talent Project and KSCX2-YW-R-116 and KSCX1-YW-R-44 of Chinese Academy of Sciences, the Ministry of Science and Technology of China (2009CB919001) and Science and Technology Committee of Shanghai Municipality (07JC14011). We thank our colleagues Ms Yingxia Zheng and Ms Chunhua Chen for technical assistance.

\section{References}

1 Bornstein MB, Miller A, Slagle S, et al. A pilot trial of Cop 1 in exacerbating-remitting multiple sclerosis. $N$ Engl J Med 1987; 317:408-414.

2 Teitelbaum D, Milo R, Arnon R, Sela M. Synthetic copolymer 1 inhibits human T-cell lines specific for myelin basic protein. Proc Natl Acad Sci USA 1992; 89:137-141.

3 Canter MB. Reflections on receiving an award. Am Psychol 2000; 55:1304-1311.

4 Zhang M, Chan CC, Vistica B, Hung V, Wiggert B, Gery I. Copolymer 1 inhibits experimental autoimmune uveoretinitis. J Neuroimmunol 2000; 103:189-194.

5 Arnon R, Aharoni R. Mechanism of action of glatiramer acetate in multiple sclerosis and its potential for the development of new applications. Proc Natl Acad Sci USA 2004; 101 (Suppl 2):14593-14598.

6 Horani A, Muhanna N, Pappo O, et al. Beneficial effect of glatiramer acetate (Copaxone) on immune modulation of experimental hepatic fibrosis. Am J Physiol Gastrointest Liver Physiol 2007; 292:G628-G638.

7 Fridkis-Hareli M, Teitelbaum D, Gurevich E, et al. Direct binding of myelin basic protein and synthetic copolymer 1 to class II major histocompatibility complex molecules on living antigen-presenting cells - specificity and promiscuity. Proc Natl Acad Sci USA 1994; 91:4872-4876.

8 Vieira PL, Heystek HC, Wormmeester J, Wierenga EA, 
Kapsenberg ML. Glatiramer acetate (copolymer-1, copaxone) promotes Th2 cell development and increased IL-10 production through modulation of dendritic cells. J Immunol 2003; 170:4483-4488.

9 Weber MS, Prod'homme T, Youssef S, et al. Type II monocytes modulate T cell-mediated central nervous system autoimmune disease. Nat Med 2007; 13:935-943.

10 Billiard F, Litvinova E, Saadoun D, et al. Regulatory and effector $\mathrm{T}$ cell activation levels are prime determinants of in vivo immune regulation. J Immunol 2006; 177:2167-2174.

11 Piccirillo CA, Tritt M, Sgouroudis E, Albanese A, Pyzik M, Hay V. Control of type 1 autoimmune diabetes by naturally occurring CD4+CD25+ regulatory T lymphocytes in neonatal NOD mice. Ann N Y Acad Sci 2005; 1051:72-87.

12 Vukmanovic-Stejic M, Zhang Y, Cook JE, et al. Human CD4+ CD25hi Foxp3+ regulatory $\mathrm{T}$ cells are derived by rapid turnover of memory populations in vivo. J Clin Invest 2006; 116:2423-2433.

13 Brunkow ME, Jeffery EW, Hjerrild KA, et al. Disruption of a new forkhead/winged-helix protein, scurfin, results in the fatal lymphoproliferative disorder of the scurfy mouse. Nat Genet 2001; 27:68-73.

14 Hori S, Nomura T, Sakaguchi S. Control of regulatory T cell development by the transcription factor Foxp3. Science 2003; 299:1057-1061.

15 Fontenot JD, Gavin MA, Rudensky AY. Foxp3 programs the development and function of CD4+CD25+ regulatory T cells. Nat Immunol 2003; 4:330-336.

16 Khattri R, Cox T, Yasayko SA, Ramsdell F. An essential role for Scurfin in CD4+CD25+ T regulatory cells. Nat Immunol 2003; 4:337-342.

17 Hong J, Li N, Zhang X, Zheng B, Zhang JZ. Induction of CD4+CD25+ regulatory $\mathrm{T}$ cells by copolymer-I through activation of transcription factor Foxp3. Proc Natl Acad Sci USA 2005; 102:6449-6454.

18 Jee Y, Piao WH, Liu R, et al. CD4(+)CD25(+) regulatory $\mathrm{T}$ cells contribute to the therapeutic effects of glatiramer acetate in experimental autoimmune encephalomyelitis. Clin Immunol 2007; 125:34-42.

19 Tisch R, McDevitt H. Insulin-dependent diabetes mellitus. Cell 1996; 85:291-297.

20 Liu E, Eisenbarth GS. Type 1A diabetes mellitus-associated autoimmunity. Endocrinol Metab Clin North Am 2002; 31:391-410, vii-viii.

21 Weiner HL. Oral tolerance with copolymer 1 for the treatment of multiple sclerosis. Proc Natl Acad Sci USA 1999; 96:33333335 .
22 Brode S, Raine T, Zaccone P, Cooke A. Cyclophosphamideinduced type- 1 diabetes in the NOD mouse is associated with a reduction of CD4+CD25+Foxp3+ regulatory T cells. $J$ Immunol 2006; 177:6603-6612.

23 Salomon B, Lenschow DJ, Rhee L, et al. B7/CD28 costimulation is essential for the homeostasis of the CD4+CD25+ immunoregulatory $\mathrm{T}$ cells that control autoimmune diabetes. Immunity 2000; 12:431-440.

24 Gregori S, Giarratana N, Smiroldo S, Adorini L. Dynamics of pathogenic and suppressor T cells in autoimmune diabetes development. J Immunol 2003; 171:4040-4047.

25 de la Rosa M, Rutz S, Dorninger H, Scheffold A. Interleukin-2 is essential for CD4+CD25+ regulatory T cell function. Eur J Immunol 2004; 34:2480-2488.

26 Thornton AM, Donovan EE, Piccirillo CA, Shevach EM. Cutting edge: IL-2 is critically required for the in vitro activation of CD4+CD25+ T cell suppressor function. J Immunol 2004; 172:6519-6523.

27 Thornton AM, Piccirillo CA, Shevach EM. Activation requirements for the induction of CD4+CD25+ T cell suppressor function. Eur J Immunol 2004; 34:366-376.

28 Chen W, Jin W, Hardegen N, et al. Conversion of peripheral $\mathrm{CD} 4+\mathrm{CD} 25-$ naive $\mathrm{T}$ cells to $\mathrm{CD} 4+\mathrm{CD} 25+$ regulatory $\mathrm{T}$ cells by TGF-beta induction of transcription factor Foxp3. J Exp Med 2003; 198:1875-1886.

29 Fantini MC, Becker C, Monteleone G, Pallone F, Galle PR, Neurath MF. Cutting edge: TGF-beta induces a regulatory phenotype in CD4+CD25- T cells through Foxp3 induction and down-regulation of Smad7. J Immunol 2004; 172:5149-5153.

30 Marie JC, Letterio JJ, Gavin M, Rudensky AY. TGF-beta1 maintains suppressor function and Foxp3 expression in CD4+CD25+ regulatory T cells. J Exp Med 2005; 201:10611067.

31 Wang Z, Hong J, Sun W, et al. Role of IFN-gamma in induction of Foxp3 and conversion of CD4+ CD25- T cells to CD4+ Tregs. J Clin Invest 2006; 116:2434-2441.

32 Skapenko A, Kalden JR, Lipsky PE, Schulze-Koops H. The IL-4 receptor alpha-chain-binding cytokines, IL-4 and IL13 , induce forkhead box $\mathrm{P} 3$-expressing $\mathrm{CD} 25+\mathrm{CD} 4+$ regulatory $\mathrm{T}$ cells from CD25-CD4+ precursors. J Immunol 2005; 175:6107-6116.

33 Maerten P, Shen C, Bullens DM, et al. Effects of interleukin 4 on $\mathrm{CD} 25+\mathrm{CD} 4+$ regulatory $\mathrm{T}$ cell function. J Autoimmun 2005; 25:112-120.

34 Hamilton-Williams EE, Palmer SE, Charlton B, Slattery RM. Beta cell MHC class I is a late requirement for diabetes. Proc Natl Acad Sci USA 2003; 100:6688-6693. 